LAWRENCE LIVERMORE NATIONAL LABORATORY

G. J. Burke

E. K. Miller

A. J. Poggio

June 2004

2004 IEEE AP-S International Symposium and USNC / URSI National Radio Science

Monterey, California

June $20-25,2004$ 
This document was prepared as an account of work sponsored by an agency of the United States Government. Neither the United States Government nor the University of California nor any of their employees, makes any warranty, express or implied, or assumes any legal liability or responsibility for the accuracy, completeness, or usefulness of any information, apparatus, product, or process disclosed, or represents that its use would not infringe privately owned rights. Reference herein to any specific commercial product, process, or service by trade name, trademark, manufacturer, or otherwise, does not necessarily constitute or imply its endorsement, recommendation, or favoring by the United States Government or the University of California. The views and opinions of authors expressed herein do not necessarily state or reflect those of the United States Government or the University of California, and shall not be used for advertising or product endorsement purposes.

This work was performed under the auspices of the U.S. Department of Energy by University of California, Lawrence Livermore National Laboratory under Contract W-7405-Eng-48. 


\title{
The Numerical Electromagnetics Code (NEC) - A Brief History
}

\author{
Gerald J. Burke* Edmund K. Miller Andrew J. Poggio* \\ * Lawrence Livermore National Laboratory \\ Los Alamos National Laboratory (Retired)
}

\section{Introduction}

The Numerical Electromagnetics Code, NEC as it is commonly known, continues to be one of the more widely used antenna modeling codes in existence. With several versions in use that reflect different levels of capability and availability, there are now 450 copies of NEC4 and 250 copies of NEC3 that have been distributed by Lawrence Livermore National Laboratory to a limited class of qualified recipients, and several hundred copies of NEC2 that had a recorded distribution by LLNL. These numbers do not account for numerous copies (perhaps 1000s) that were acquired through other means capitalizing on the open source code, the absence of distribution controls prior to NEC3 and the availability of versions on the Internet.

In this paper we briefly review the history of the code that is concisely displayed in Figure 1. We will show how it capitalized on the research of prominent contributors in the early days of computational electromagnetics, how a combination of events led to the tri-service-supported code development program that ultimately led to NEC and how it evolved to the present day product.

The authors apologize that space limitations do not allow us to provide a list of references or to acknowledge the numerous contributors to the code both of which can be found in the code documents.

\section{The Early History}

The 1960s ushered in the serious use of digital computers for the solution of boundary value problems using integral equations. The publications of Roger Harrington, Jack Richmond and Ken Mei provided substantial background and indications of what could be done. Interest in applications was growing as evidenced by the expanding support for research in the area by the US government and by published applications by commercial entities such as Granger Associates, TCI, Inc. (Andreasen, Harris, Tanner), and MBAssociates (Bosserman and Burke). The genesis for NEC basically begins at this point.

In the mid-1960s, MBAssociates, a small firm in San Ramon, California was designing and manufacturing small radar chaff dispensers and needed an ability to predict the radar cross section of the decoys. Homer Bosserman of MBA, aware of the work of Jack Richmond (1965), acquired the Ohio State code (pulse basis functions, point matching, MoM solver of Pocklington's Integral Equation for conducting wires) and began using it for loops and straight wires. Dr. Bosserman sought to improve the performance of the code so he and Jerry Burke introduced the three-term basis function (constant plus sine plus cosine) that had been developed and used by Ken Mei (1965) in his work with point matching solutions of Hallen's Integral Equation for straight and curved wires. The combination, developed with support from the Air Force Space and Missile Systems Organization, was referred to as BRACT and circa 1967 became the workhorse at MBAssociates for scattering calculations. In 1968 a version referred to as ANTBRACT was developed for antenna calculations by introducing voltage sources on the wires and the Reflection Coefficient Approximation for grounds.

The bellwether in the development of a user-oriented electromagnetic modeling tool for antennas and scattering took place in 1970 when the US government formalized its interest in a user-oriented tool capable of antenna calculations. A Request for Proposal, jointly supported by the Naval Research laboratory, the Naval Ship Engineering Center, the US Army ECOM/Communication Systems, the US Army Strategic Communications 
Command and US Air Force Rome Air Development Center, was issued and competed. In the eyes of the MBA team that at that time included the authors, the competition included the prolific university researchers who had produced significant foundations of mathematics and algorithms that enabled a strong modeling capability. It also included a very qualified team from TCI, Inc. that had evolved from TRG and Granger Associates and included Mogens Andreasen, Frank Harris and R. L. Tanner. TCI had a very capable antenna modeling tool that was being used in support of the design and development of their commercial products. The computer program was proprietary but presentations by Dr. Andreasen permitted glimpses of the algorithms and I/O data formats. Their tool, like the university research tools and ANTBRACT, had state-of-the-art modeling capabilities but also showed signs of user-friendly input and output formats. The latter motivated others to make the needed improvements in I/O capabilities.

The proposal competition was concluded in 1971 and the multi-sponsor award was ultimately made to MBAssociates in mid-1971.

\section{The Evolution of the Antenna Modeling Program (AMP)}

The tri-service project at MBAssociates was focused on the refinement of ANTBRACT and the preparation of user manuals. The code was called AMP (Antenna Modeling Program). By July 1972 the code and the documentation, consisting of an Engineering Manual detailing theory and techniques, a Systems Manual describing the specifics in the code, and a User's Manual providing machine-specific guidance for running the code, was complete. AMP was implemented at each of the sponsoring agencies on computers such as the CDC 3800, 6500 and 6700, the GE645 and the Burroughs B5700. During this initial phase of refinement and delivery to the user community AMP was championed and evaluated by many including Messrs. Malagisi and Potenza of RADC; Musselman, Royce and McGown of NRL; Singer, Pride, Ogelsby and Mink of NAVSEC; Krensky, Berinstein and Warshauer of ECOM/CSA, and Bramel and Lane of STRATCOM.

No sooner was AMP "completed" than the sponsors expressed a desire for improved computer runtimes for problems that included complex wire antennas and large voluminous structures with wire appendages. Of course, AMP was already being used for modeling voluminous bodies using wire grids but such modeling was proving to be time-intensive so a reduction was sought by using the thin wire EFIE for the wires and the magnetic field integral equation (MFIE) for the voluminous structures. Albertson, Hansen and Jensen were already using the MFIE for antennas on solids while Oshiro and Mitzner were using it for RCS. To improve runtimes for wire grid modeling, an option was introduced for using approximations for the matrix elements when source and observation point separation exceeded a specified value. The modified code was referred to as AMP2 and a supplementary computer program manual was appended to the AMP documentation. The techniques and timesaving were demonstrated by the analysis of wire antenna systems on a US Navy frigate (1975).

\section{And then it became NEC}

By 1977, the Air Force Weapons Lab (Phil Castillo), focused on EMP effects, and the Naval Ocean Systems Center (John Rockway), focused on shipboard communication systems, became the prime drivers behind AMP. They suggested improvements in the code, namely, an improved current basis function and an extended thin wire kernel. In view of these changes, Carl Baum suggested that AMP become the Numerical Electromagnetics Code (NEC) and both he and John Rockway suggested that it be a part of a broader suite for evaluating electromagnetic interactions over a very broad frequency range. The framework was meant to include the NEC being discussed here as 'NEC Method of Moments' for structures that were a few wavelengths in size and other elements to enable a capability for large structures (in terms of wavelengths) such as might be modeled using asymptotic techniques. In fact, John Rockway promoted the inclusion of an element referred to as 'NEC - Basic Scattering Code' that appended the Ohio State University High Frequency Scattering Code based on the Uniform Theory of 
Diffraction to the NEC suite to provide a broad-based capability. This latter code, commonly referred to as NEC-BSC, has also been widely used.

The next revision of NEC responded to a requirement for accurate modeling of wire antennas in close proximity to realistic grounds and for a capability that enabled what-if siting studies of antennas on large bodies such as ships. The former requirement was met by using Sommerfeld Integrals for the interactions and Interpolation of these quantities over spatial coordinates to provide accuracy and reasonable runtimes for wire antennas above ground. The interpolation was an example of what later become known as ModelBased Parameter Estimation. The latter requirement was met by using matrix partitioning schemes that minimized repeated interaction calculations when portions of the problem space were invariant during the geometry perturbations. A User Guide and a Program Description were published. This version, NEC2, was released in 1980.

Within a few years a critical deficiency became apparent. NEC2 could not be used for modeling buried wires because it required the wire structure to be in free space or in the lossless half-space with $\varepsilon_{\mathrm{r}}=1$ above a finitely conducting ground. The US Army/ISEC joined NOSC in funding an effort to rectify this deficiency. In 1983 a new version NEC3, with appropriate documentation, was released. In addition to the above-mentioned capabilities, NEC3 also enabled modeling of wires and surfaces (using the MFIE) in infinite dielectric or lossy media. The US government, sensitive to national security interests, viewed NEC3 as military critical and limited NEC3 distribution to appropriately qualified organizations. Even with this restriction, 250 copies were distributed.

The next chapter in the NEC history came with the release of NEC4 in 1990. This version opened the door to computations at extremely low frequencies. With the support of NOSC (Rockway, Logan and Li) and the Army/ISEC (J. McDonald and R. Franklin), NEC4 overcame these difficulties and added capabilities for accurately modeling wires of stepped radius and insulated wires.

\section{NEC - A Living Thing}

NEC has had a history in various incarnations that exceeds 30 years. Yet, it continues to be a code-in-demand with a wide-based user group for reasons that extend beyond the simple "it's a high quality product with a detailed set of manuals representing an open source code". NEC adds another dimension to the user-code world - user engagement.

The open source has allowed users to get inside the code, if desired, to modify and tweak. But that's not all. The users have formed a user fraternity that is a massive informationsharing network that communicates very effectively using the Internet. At present there are websites that feature NEC, e.g., the Numerical Electromagnetics Code (Method of Moments) website hosted by Trevor Marshall at http://www.nec2.org/ that provides tutorials, links to information and instructions on running NEC2 in a Windows environment, the NEC2 documentation, and links to other sites where one can obtain NEC for other operating systems. There is also an active mailing list (neclist@gweep.ca) that is meant for discussion of computational EM issues and is a major platform for NEC discussions, theoretical and operational.

A major factor in NEC's success is the fact that it has been supported over the years. A code cannot survive without support, maintenance and troubleshooting. Having an expert closely tied to the code over its history who is able to deal with technical and operational issues is critical to longevity. NEC has been uniquely fortunate in this respect. 


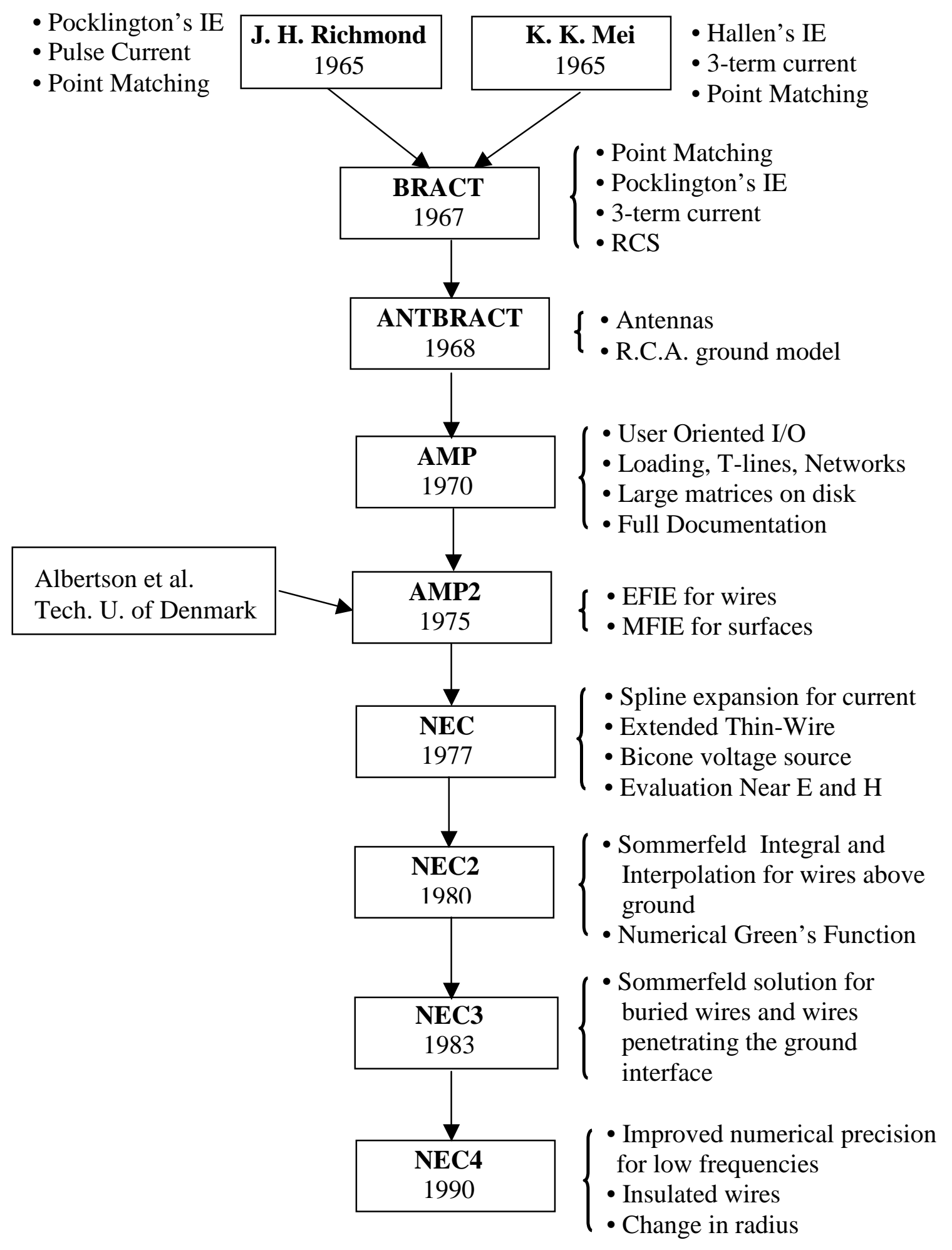

Figure 1. Genesis 